Gross, J. (1956). J. biophys. biochem. Cytol. 2, 261.

Gross, J. (1958). J. exp. Med. 108, 215.

Gross, J., Highberger, J. H. \& Schmitt, F. O. (1955). Proc. nat. Acad. Sci., Wash., 41, 1.

Gross, J. \& Kirk, D. (1958). J. biol. Chem. 233, 355.

Hall, C. E. (1956). Proc. nat. Acad. Sci., Wash., 42, 801.

Hall, C. E. \& Doty, P. (1958). J. Amer. chem. Soc. 80, 1269.

Hawn, C. v. Z. \& Porter, K. R. (1947). J. exp. Med. 86, 285.

Jackson, D. S. (1958a). Recent Advances in Gelatin and Glue Research, p. 50. Ed. by Stainsby, G. London: Pergamon Press Ltd.

Jackson, D. S. (1958b). New. Engl. J. Med. 259, 514.

Jackson, D. S. \& Fessler, J. H. (1955). Nature, Lond., 176, 69.

Jackson, D. S. \& Neuberger, A. (1957). Biochim. biophys. Acta, 26, 638.

Jackson, S. F. (1954a). Nature, Lond., 173, 950.

Jackson, S. F. (1954b). Proc. Roy. Soc. B, 142, 536.

Jackson, S. F. (1955). Proc. Roy. Soc. B, 144, 556.

Jackson, S. F. (1956). Proc. Roy. Soc. B, 146, 270.

King, E. J. (1932). Biochem. J. 26, 292.

Lowry, O. H., Rosebrough, N. J., Farr, A. I. \& Randall, R. J. (1951). J. biol. Chem. 198, 265.

Mathews, M. B., Kulonen, E. \& Dorfman, A. (1954). Arch. Biochem. Biophys. 52, 247.

Meyer, K. (1946). Amer. J. Med. 1, 675.

Meyer, K. (1955-56). Harvey Lect. 1, 97.

Neuman, R. E. \& Logan, M. A. (1950). J. biol. Chem. 184, 299.

Biochem. $J$. (1960) 75, 598

\title{
The Formation of Fibrils from Collagen Solutions
}

\section{A MECHANISM OF COLLAGEN-FIBRIL FORMATION}

\author{
By G. C. WOOD \\ Nuffield Gerontological Research Unit, Department of Medicine, University of Leeds
}

(Received 22 June 1959)

In the preceding paper (Wood \& Keech, 1960) evidence was adduced for the hypothesis that precipitation of collagen fibrils from collagen solutions occurs in two steps, the first occurring during the lag period, the second being represented by the sigmoid precipitation curve. This suggestion has also been put forward by Bensusan \& Hoyt (1958). The ultimate fibril width appears to be determined during the first step. It is the purpose of the present paper to see how the experimental results may be interpreted quantitatively in terms of a two-step mechanism.

Freshly prepared collagen precipitates can be redissolved by altering the $\mathrm{pH}$ or temperature and then reprecipitated by returning these variables to their original values (Jackson, 1957; Gross, 1958).
It seems reasonable therefore to regard the formation of collagen fibrils as precipitation of the protein, in an ordered form, from solution in which it is supersaturated under the particular conditions of $\mathrm{pH}$, ionic strength, temperature, etc. One might expect therefore some similarity between the precipitation of collagen and the precipitation of sparingly soluble inorganic salts, in crystalline form, from supersaturated solution.

The kinetics of the latter process has been widely studied and in many cases it has been recognized that the process takes place in two steps: $(a)$ aggregation of the particles in solution to form nuclei, i.e. clusters of particles of just sufficient size to be stable as a separate phase: (b) growth of these nuclei, by accretion of soluble material, to 
form a visible precipitate. Equations describing the kinetics of these two steps have been derived and applied with some success to a number of systems (e.g. Johnson \& O'Rourke, 1954; Duke \& Brown, 1954; Nielsen, 1955, 1958; Collins \& Leinweber, 1956).

The mechanism of protein precipitation is probably a good deal more complicated than that of inorganic salts. This is likely to be particularly so in the formation of collagen fibrils, where highly asymmetric soluble particles aggregate in a very specific manner with their axes parallel to the ultimate fibril axis (Schmitt, Gross \& Highberger, $1955 a, b$; Gross, 1956; Schmitt, 1959). Nevertheless, the same general principles should be applicable. Waugh (1957) has, in fact, applied the nucleation-growth concept to the precipitation of insulin fibrils, which, like collagen fibrils, are the result of aggregation of asymmetric particles.

It is of interest therefore to see how far the idea accounts for the rate of formation of collagen fibrils.

Following Waugh's treatment of the problem, the rate of formation of nuclei,

$$
\frac{\mathrm{d} n}{\mathrm{~d} t}=k_{1} C^{x}
$$

where $C$ is concentration of collagen, $n$ is concentration of nuclei, $x$ is a constant and $k_{1}$ is a rate constant. This equation implies that $x$ collagen particles must come together simultaneously to form a nucleus. The rate of growth of each precipitated particle,

$$
\frac{\mathrm{d} m}{\mathrm{~d} t}=k_{2} A C,
$$

where $m$ is mass of the precipitated particle, $A$ is aggregating area and $k_{2}$ is a rate constant. $A$ is assumed to be proportional to the surface area and the further assumption is made that the shape of the precipitated particle remains unchanged during its growth. Then $A \propto \mathrm{m}^{\text {s }}$, and equation (2) becomes

$$
\frac{\mathrm{d} m}{\mathrm{~d} t}=k_{3} m^{3} C \text {. }
$$

The rate of growth is thus assumed to be controlled by the condensation of the dissolved collagen on to the precipitate and not by its diffusion to the surface of the precipitated particles.

If the growth process is reversible, equation (3) must be modified to

$$
\frac{\mathrm{d} m}{\mathrm{~d} t}=k_{3} m^{\frac{\mathrm{g}}{3}}\left(C-C_{s}\right),
$$

where $C_{s}$ is the value of $C$ at equilibrium, i.e. the solubility of the collagen.

In the very early stages of precipitation, when
$C \simeq C_{0}\left(C_{0}\right.$, initial collagen concentration) equation (4) may be integrated:

$$
m=\frac{k_{3}^{3}\left(C_{0}-C_{s}\right) t^{3}}{27}
$$

Introducing equation (1),

$$
m \mathrm{~d} n=\frac{k_{1} k_{3}^{3} C_{0}^{x}\left(C_{0}-C_{s}\right)^{3} t^{3}}{27} \mathrm{~d} t
$$

therefore

$$
\left(C_{0}-C\right)=\int_{0}^{n_{t}} m \mathrm{~d} n=\frac{k_{1} k_{3}^{3} C_{0}^{x}\left(C_{0}-C_{s}\right)^{3} t^{4}}{108} .
$$

Introducing the extent of precipitation,

$$
p=\left(C_{0}-C\right) /\left(C_{0}-C_{s}\right),
$$

and putting $\left(C_{0}-C_{s}\right) / C_{0}=\beta$,

$$
p=\frac{K_{1} \beta^{2} C_{0}^{(x+2)}}{108} t^{4}
$$

where

$$
K_{1}=k_{1} k_{3}^{3} \text {. }
$$

This relationship will clearly result in an apparent lag period such as is actually observed (Bensusan \& Hoyt, 1958; Wood \& Keech, 1960).

As the collagen concentration falls appreciably below $C_{0}$ the rate of nucleation as given by equation (1) falls rapidly due to the term $C^{x}$. At the same time the rate of growth of each precipitated particle as given by equation (4) increases as the particle grows, due to the term $m^{\text {s }}$, until eventually the fall of collagen concentration slows growth down too. Thus the major part of precipitation will occur by growth of those nuclei formed early in the reaction, i.e. during the lag period. The contribution due to growth of nuclei formed later will be small, its actual extent depending on the relative values of $k_{1}, k_{3}$ and $x$. Assuming that their contribution is negligible, we have at the end of the lag period a set of small particles whose subsequent growth is responsible for all further precipitation.

It can now be seen how the observed two-step nature of precipitation (Wood \& Keech, 1960) arises in terms of this mechanism. The first step, occupying the lag period, consists mainly of the formation of nuclei whereas the second consists of the growth of these nuclei into fibrils. The fibrillar nature of the precipitate results from the asymmetry of the nuclei and of the collagen macromolecules themselves. In the closed system studied experimentally the size of the fibrils is determined by the number and the shape of the nuclei and is hence decided during the lag period. It is not affected by change in rate of growth after the lag period, provided that the manner in which growth takes place does not change at the same time. The mechanism thus accounts qualitatively for the observation that although precipitation is accelerated by increasing the temperature either before or 
after the lag period, fibril width is altered only if the temperature is increased before the lag period and is not affected by increasing the temperature after the lag period.

For low values of $p$ the predicted course of precipitation is given by equation (6). The size distribution and hence the growth characteristics, at the end of the lag period, of those particles formed during the lag period might be calculated from equation (5) and the integrated form of equation (1):

$$
n=k_{1} C_{0}^{x} t .
$$

The time course of precipitation after the $\operatorname{lag}_{1}$,riod could then be calculated by summing the contribution of all the species of particle. The calculation can be simplified by replacing the distribution of particles by a concentration, $n_{e}$, of hypoth tical identical particles. The value of $n_{e}$ and the size of these particles is determined by the conditions that their total surface area and total mass must be the same as those of the actual distribution of particles. Then from equation (4)

$$
n_{e} \frac{\mathrm{d} m}{\mathrm{~d} t}=k_{3} n_{e} m^{\frac{2}{3} C},
$$

which may be transformed into

$$
\frac{\mathrm{d} p}{\mathrm{~d} t}=K_{2} \beta^{\frac{2}{3}} C_{0}^{2} p^{\frac{z}{z}}(1-p),
$$

where $K_{2}=k_{3} n_{e}^{\frac{1}{t}}$. On integration this becomes:

$$
K_{2} \beta^{\frac{2}{3}} C_{0}^{\frac{2}{2}} t=\int \frac{\mathrm{d} p}{p^{\frac{2}{3}}(1-p)}=\mathbf{I},
$$

where

$$
I=\frac{1}{2} \ln \left\{\frac{R^{2}+R+1}{(R-1)^{2}}\right\}+\sqrt{ } 3 \tan ^{-1}\left\{\frac{\sqrt{ } 3}{1+2 R^{-1}}\right\}
$$

and $\boldsymbol{R}=p^{k}$.

\section{Application of equations (6) and (9) to experimental data}

The experimental data are those given in the preceding paper (Wood \& Keech, 1960). In all the experiments the collagen was almost completely precipitated and therefore $\beta \simeq 1$.

Application of equation (6). Graphs of $p$ against $t^{4}$ for a series of experiments at different collagen concentrations are shown in Fig. 1. As predicted by equation (6) these are linear at low values of $p$. According to equation (6) the slopes $(S)$ are given by

$$
\log S=\log \frac{K_{1}}{108}+(x+2) \log C_{0} .
$$

$\log S$ is plotted against $\log C_{0}$ in Fig. 2. The linearity of the curve indicates that $K_{1}$ and $x$ are independent of collagen concentration and the values of the parameters calculated from the slopes and intercepts of these and other similar graphs are given in Tables 1-3. Thus is appears that equation (6) satisfactorily describes precipitation in the region of low $p$. The experimental error in measurement of $p$ in this region is, however, quite large so that the experiments do not provide a very critical test and the values of $K_{1}$ and $x$ are only approximate.

Application of equation (9). The integral $I$ is plotted in Fig. 3 as a function of $p$. By the use of this graph to obtain I from $p$, I was plotted against time $(t)$ for a set of data at different collagen concentrations (Fig. 4). As predicted by equation (9),

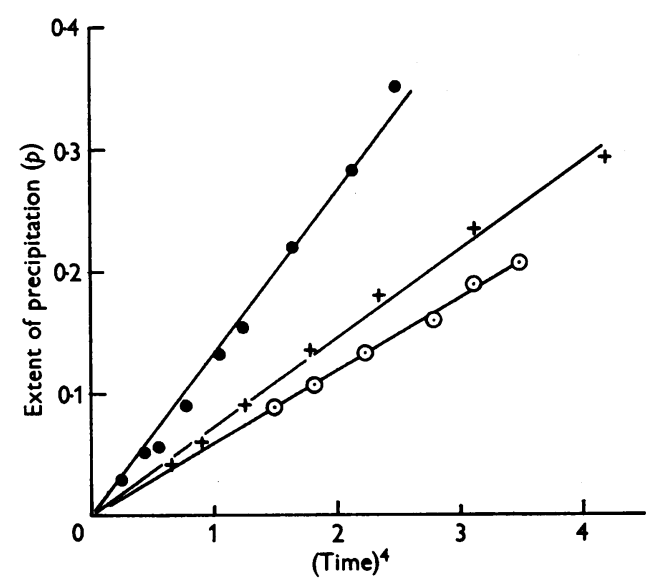

Fig. 1. Early stages of precipitation. Application of equation (6) to experimental data at $\mathrm{pH} 7 \cdot 1, I \quad 0.23$ and $25^{\circ}$. Initial collagen concn.: $\bullet, 0.084 \%$;, $0.028 \%$; $\odot$, $0.017 \%$. Units of (time) $)^{4}$ are: $0,10^{4}$ min. $^{4} ;+, 10^{6}$ min. ${ }^{4}$; $\odot, 10^{7} \min ^{4}$

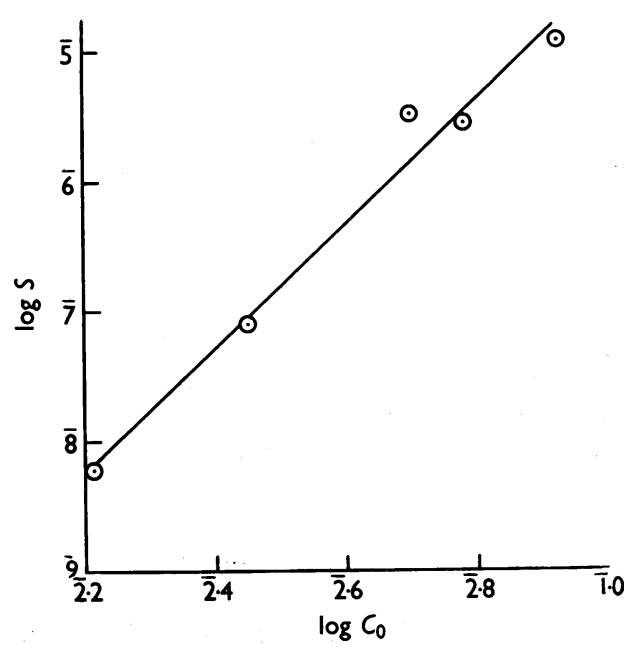

Fig. 2. Early stages of precipitation. Application of equation (6). $S$ is the slope of graph of $p$ against $t^{4}$. 
the curves are linear over most of their course. The slopes are proportional to $K_{2}$, whereas the intercepts, $t_{c}$, mark the ends of the lag periods. The deviation from linearity at low values of $p$ might be expected, due to overlap of the nucleation and growth phases, whereas deviation at values of $p$

Table 1. Effect of collagen concentration on the parameters for nucleation and growth

Experimental conditions: $25^{\circ}$; $\mathrm{pH} 7 \cdot 1 ; I$ 0.23. For definitions of $C_{0}, x, K_{1}, t_{c}$ and $K_{2}$, see text. The results are for two different preparations of collagen solution.

\begin{tabular}{|c|c|c|c|c|}
\hline $\begin{array}{c}C_{0} \\
(\%)\end{array}$ & $x$ & $K_{1}$ & $\begin{array}{c}t_{c} \\
\text { (min.) }\end{array}$ & $K_{2}$ \\
\hline $\left.\begin{array}{l}0.084 \\
0.061 \\
0.050 \\
0.028 \\
0.017\end{array}\right\}$ & $2 \cdot 7$ & 227 & $\left\{\begin{array}{r}4 \cdot 7 \\
5 \cdot 8 \\
5 \cdot 4 \\
14 \cdot 0 \\
25 \cdot 5\end{array}\right.$ & $\begin{array}{l}1.49 \\
1.10 \\
1.32 \\
0.76 \\
0.49\end{array}$ \\
\hline $\left.\begin{array}{r}0.050 \\
0.034 \\
0.017\end{array}\right\}$ & 2.5 & 100 & $\left\{\begin{array}{r}3 \cdot 8 \\
7 \cdot 2 \\
14 \cdot 5\end{array}\right.$ & $\begin{array}{l}0.99 \\
0.58 \\
0.49\end{array}$ \\
\hline
\end{tabular}

Table 2. Effect of $\mathrm{pH}$ and ionic strength on the parameters for nucleation and growth

Experimental conditions: $25^{\circ} ; C_{0} 0.05 \%$. For definitions of $x, K_{1}, t_{c}$ and $K_{2}$, see text. Each set of results was obtained on a different preparation of collagen solution.

\begin{tabular}{|c|c|c|c|c|c|}
\hline $\mathrm{pH}$ & $I$ & $x$ & $K_{1}$ & $\begin{array}{c}t_{c} \\
(\min .)\end{array}$ & $K_{2}$ \\
\hline $\begin{array}{l}6 \cdot 0 \\
7 \cdot 1 \\
7 \cdot 5 \\
8 \cdot 0\end{array}$ & $\begin{array}{l}0 \cdot 23 \\
0 \cdot 23 \\
0 \cdot 23 \\
0 \cdot 23\end{array}$ & $\begin{array}{l}- \\
z\end{array}$ & E & $\begin{array}{l}2 \cdot 0 \\
7 \cdot 0 \\
7 \cdot 5 \\
6 \cdot 8\end{array}$ & $\begin{array}{l}4 \cdot 42 \\
1 \cdot 03 \\
0.96 \\
1 \cdot 33\end{array}$ \\
\hline $\begin{array}{l}6 \cdot 0 \\
7 \cdot 1 \\
8 \cdot 0\end{array}$ & $\begin{array}{l}0 \cdot 23 \\
0 \cdot 23 \\
0 \cdot 23\end{array}$ & $\begin{array}{l}2 \cdot 5 \\
2 \cdot 7 \\
3 \cdot 0\end{array}$ & $\begin{array}{r}30500 \\
1400 \\
3100\end{array}$ & $\begin{array}{l}2 \cdot 0 \\
5 \cdot 0 \\
4 \cdot 7\end{array}$ & $\begin{array}{l}6 \cdot 12 \\
1 \cdot 77 \\
2 \cdot 28\end{array}$ \\
\hline $\begin{array}{l}7 \cdot 0 \\
7 \cdot 0 \\
7 \cdot 0\end{array}$ & $\begin{array}{l}0 \cdot 13 \\
0 \cdot 23 \\
0 \cdot 31\end{array}$ & 二 & E & $\begin{array}{r}1.0 \\
2.5 \\
26 \cdot 3\end{array}$ & $\begin{array}{l}7 \cdot 44 \\
1 \cdot 18 \\
0 \cdot 45\end{array}$ \\
\hline $\begin{array}{l}7 \cdot 0 \\
7 \cdot 0 \\
7 \cdot 0\end{array}$ & $\begin{array}{l}0 \cdot 13 \\
0 \cdot 23 \\
0 \cdot 31\end{array}$ & $\begin{array}{l}1 \cdot 8 \\
1 \cdot 8 \\
3 \cdot 3\end{array}$ & $\begin{array}{r}68000 \\
1400 \\
540\end{array}$ & $\begin{array}{r}1.0 \\
5 \cdot 0 \\
10 \cdot 0\end{array}$ & $\begin{array}{l}9 \cdot 28 \\
1 \cdot 77 \\
0 \cdot 81\end{array}$ \\
\hline $\begin{array}{l}6 \cdot 0 \\
6 \cdot 0 \\
6 \cdot 0\end{array}$ & $\begin{array}{l}0 \cdot 13 \\
0 \cdot 23 \\
0 \cdot 31\end{array}$ & E & 二 & $\begin{array}{l}1.0 \\
2 \cdot 0 \\
1.0\end{array}$ & $\begin{array}{l}9 \cdot 36 \\
4 \cdot 22 \\
7 \cdot 22\end{array}$ \\
\hline $\begin{array}{l}8 \cdot 0 \\
8 \cdot 0 \\
8 \cdot 0\end{array}$ & $\begin{array}{l}0 \cdot 13 \\
0 \cdot 23 \\
0 \cdot 31\end{array}$ & E & 二 & $\begin{array}{r}3 \cdot 1 \\
6 \cdot 8 \\
27 \cdot 0\end{array}$ & $\begin{array}{l}4 \cdot 57 \\
1 \cdot 32 \\
0 \cdot 48\end{array}$ \\
\hline
\end{tabular}

Table 3. Effect of temperature on the parameters for nucleation and growth

Experimental conditions: $\mathrm{pH} 7 ; I 0 \cdot 23 ; C_{0} 0.05 \%$. For definitions of $x, K_{1}, K_{2}$ and $\left(K_{2}\right)_{g}$ see text.

$\begin{array}{ccrrr}\text { Temp. } & x & K_{1} & K_{2} & \left(K_{2}\right)_{g} \\ 20^{\circ} & - & - & 0.18 & 0.17 \\ 25 & 2.5 & 100 & 0.99 & 1.02 \\ 30 & 1.9 & 440 & 2 \cdot 25 & 1.55 \\ 37 & 1.6 & 28000 & 11.35 & 4.85\end{array}$

approaching unity might be due to the sensitivity of $I$ to errors in $p$ in this region. In spite of these deviations equation (9) describes the shape of the precipitation curves quite well, as shown in Fig. 5, where the smooth curves were calculated from values of $K_{2}$ and $t_{c}$ obtained from straight lines such as those shown in Fig. 4. Similar results were obtained for a wide range of conditions and the values of $K_{2}$ and $t_{c}$ are given in Tables 1-3.

\section{Fibril width}

The successful application of equation (9) to precipitation curves and the fact that the mechanism accounts qualitatively for the observation that fibril size is determined during the lag period suppbrts the assumption that only those nuclei formet during the lag period contribute significantly to precipitation.

According to the simple mechanism, at any stage of precipitation these nuclei will have grown into

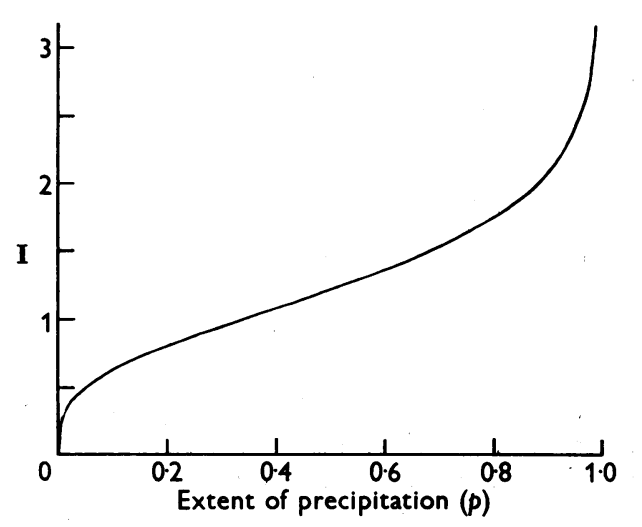

Fig. 3. The integral I plotted as a function of $p$ (see equation 9).

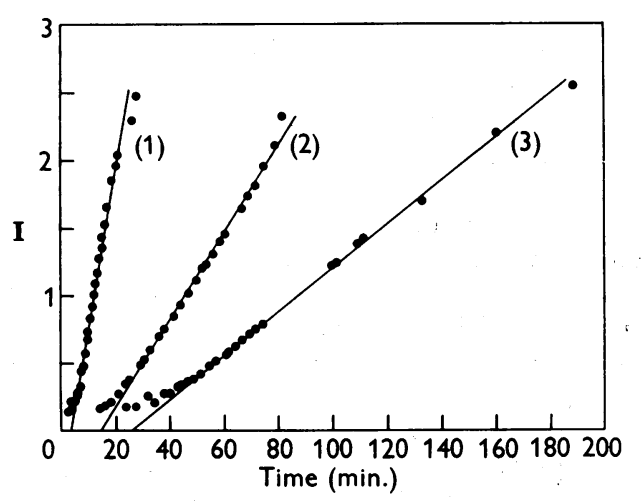

Fig. 4. Precipitation at $\mathrm{pH} 7 \cdot 1, I 0.23$ and $25^{\circ}$ at three different collágen concentrations: (1) $0.084 \%$; (2) $0.028 \%$; (3) $0.017 \%$. Data are plotted in accordance with equation (9). 


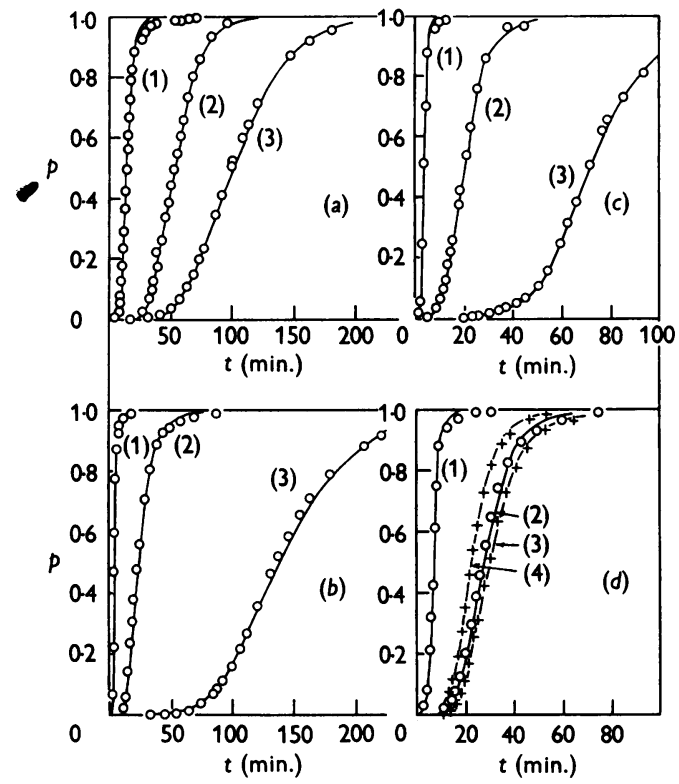

Fig. 5. Precipitation under different experimental conditions. The curves represent the best fit of equation (9) to the experimental data. (a) Different collagen concentrations $C_{0}$ : (1) $0.084 \%$; (2) $0.028 \%$; (3) $0.017 \%$. (b) Different temperatures: (1) $37^{\circ}$; (2) $25^{\circ}$; (3) $20^{\circ}$. (c) Different ionic strengths, $I$ : (1) 0.13 ; (2) 0.23 ; (3) 0.31 . (d) Different $\mathrm{pH}$ values: (1) $\mathrm{pH} 6.0$; (2) $\mathrm{pH} 7 \cdot 1$; (3) $\mathrm{pH} 7 \cdot 5$; (4) $\mathrm{pH} 8.1$. Except for the parameters stated in each set, conditions were: $\mathrm{pH} 7.1 ; I 0.23 ; C_{0} 0.05 \%$; temp. $25^{\circ}$.

fibrils, the largest of which correspond to nuclei formed at zero time and the smallest to nuclei formed at $t=t_{c}$. From equations (5) and (7), the distribution of fibril width will be such that there are equal numbers of fibrils of different widths, between the two extremes, if, as assumed earlier, the shape of the fibrils remains constant during growth. The observed distribution is narrow but does not conform precisely to this prediction (Wood \& Keech, 1960), as may be seen from the histograms reproduced in Figs. 6 and 7. Since it is likely, however, that the shape of the fibrils is distorted during drying for electron microscopy (see Anderson, 1956), this does not necessarily invalidate the assumption of constant shape and, as shown below, the observed distribution is not inconsistent with the mechanism.

From equation (4) the mass of each of the smallest fibrils $\left(m_{c}\right)$ at any time, $t$, is given approximately by

$$
\left(m_{c}\right)_{t}^{t}=\frac{k_{3}}{3} \int_{t_{0}}^{t} C \mathrm{~d} t=\frac{k_{3} C_{0}}{3} \int_{t_{c}}^{t}(1-p) \mathrm{d} t .,
$$

Regarding each fibril as a long cylinder of radius $r$ and length $l$,

$$
\left(m_{c}\right)_{t}=\rho \pi \alpha\left(r_{c}\right)_{t}^{3},
$$

where $\rho$ is density, $\alpha(=l / r)$ is a constant and $\left(r_{c}\right)_{t}$ is radius of each of the smallest fibrils at time $t$.

Therefore $\quad\left(r_{c}\right)_{t}=\frac{k_{3} C_{0}}{3(\rho \pi \alpha)^{\frac{1}{3}}} \int^{t}(1-p) \mathrm{d} t$.

Similarly, the radius $\left(r_{0}\right)$ of the largest fibrils is

$$
\begin{aligned}
& \left(r_{0}\right)_{t}= \\
& \frac{k_{3} C_{0}}{3(\rho \pi \alpha)^{\frac{3}{3}}} \int_{0}^{t}(1-p) \mathrm{d} t=\frac{k_{3} C_{0}}{3(\rho \pi \alpha)^{\frac{3}{3}}}\left\{t_{c}+\int_{t_{c}}^{t}(1-p) \mathrm{d} t\right\} .
\end{aligned}
$$

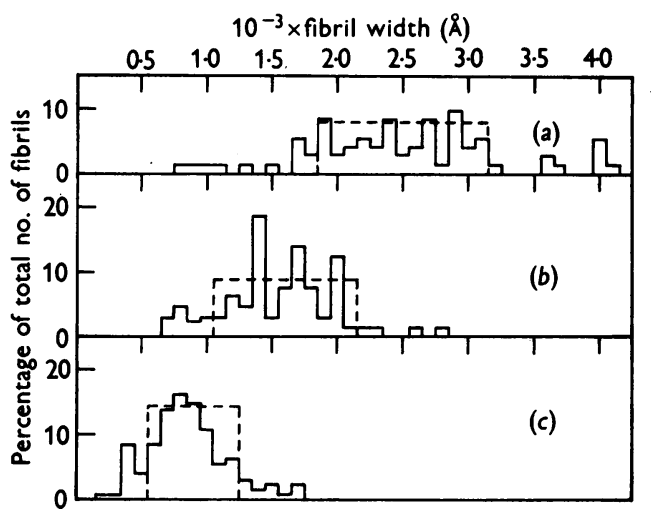

Fig. 6. Comparison of calculated (broken lines) and observed (full lines) fibril-width distributions. Data were obtained at $\mathrm{pH} 7 \cdot 1, C_{0} 0.05 \%$ and $25^{\circ}$. Ionic strengths, $I$ : (a) $0.31 ;(b) 0.23$; (c) 0.13 .

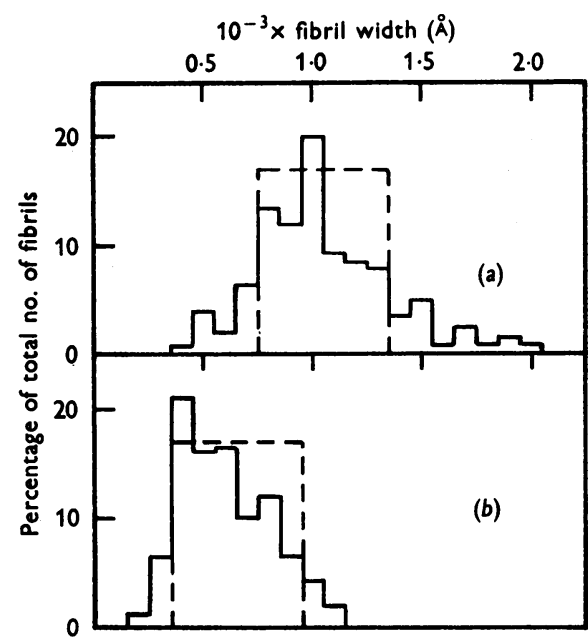

Fig. 7. Comparison of calculated (broken lines) and observed (full lines) fibril-width distributions. Data were obtained at pH $7 \cdot 1, I 0.23 ; C_{0} 0.05 \%$. Temperatures: (a) $25^{\circ}$; (b) $37^{\circ}$. 
When precipitation is complete the ratio of the radii of the largest and smallest fibrils is

$$
\frac{\left(r_{0}\right)_{\infty}}{\left(r_{c}\right)_{\infty}}=\frac{t_{c}+\int_{t_{c}}^{\infty}(1-p) \mathrm{d} t}{\int_{t_{c}}^{\infty}(1-p) \mathrm{d} t} .
$$

The right-hand side may be computed from experimental precipitation curves and the results of doing this for several experiments are shown in Table 4. In Figs. 6 and 7 the calculated spread of fibril width is compared with the observed distribution. One limit of the calculated distribution was chosen so that the maximum number of fibrils should be included and in all cases more than $70 \%$ of the fibrils come within the predicted limits, indicating that the observed spread of fibril width is consistent with the mechanism.

\section{Separation of the nucleation and growth steps}

Each of the parameters $K_{1}$ and $K_{2}$, resulting from analysis of precipitation curves, involves constants relating to both nucleation and growth. The effect of varying experimental conditions on each of the steps cannot therefore be determined from the variation of $K_{1}$ and $K_{2}$ alone. The contribution of growth to $K_{2}$ can in principle be estimated by analysis of fibril-width distribution, to give $n_{e}$, but the existing electron-microscope data are probably not sufficiently precise. Information about the growth step can, however, be obtained by an alternative method. If, in a series of precipitations, nucleation (i.e. lag period) is allowed to proceed under identical conditions in all experiments the effect of any subsequent change of conditions must be attributed to its effect on the growth step.

Table 3 shows data for a series of experiments in which the temperature during nucleation was $25^{\circ}$ in all experiments but in which subsequent growth was allowed to proceed at different temperatures. (A typical experiment of this type was described in the preceding paper.)

\section{Table 4. Effect of ionic strength and temperature on distribution of fibril radius}

Experimental conditions: $\mathrm{pH} 7 \cdot 1 ; C_{0} 0.05 \%$. For definitions of $p, t_{c},\left(r_{0}\right)_{\infty}$ and $\left(r_{c}\right)_{\infty}$, see text. Each set of results was obtained on a different preparation of collagen solution.

$\begin{array}{ccccc}\text { Temp. } & I & \int_{t_{c}}^{\infty}(1-p) \mathrm{d} t & \begin{array}{c}t_{c} \\ (\min .)\end{array} & \frac{\left(r_{0}\right)_{\infty}}{\left(r_{c}\right)_{\infty}} \\ 25^{\circ} & 0.13 & 1.02 & 1.0 & 1.98 \\ 25 & 0.23 & 5.51 & 5.0 & 1.91 \\ 25 & 0.31 & 26.35 & 10.0 & 1.61 \\ 25 & 0.23 & 16.5 & 10.0 & 1.60 \\ 37 & 0.23 & 1.4 & 2.0 & 1.80\end{array}$

Now $K_{2}=k_{3} n_{b}^{\mathrm{t}}$, and since $n_{e}$ is constant under these conditions

$$
\frac{\mathrm{d} \ln \left(K_{2}\right)_{0}}{\mathrm{~d} 1 / T}=\frac{\mathrm{d} \ln k_{3}}{\mathrm{~d} 1 / T}=-\frac{E_{\Delta}}{R},
$$

where $\left(K_{2}\right)_{g}$ is the value of $K_{2}$ when nucleation occurs at $25^{\circ}, E_{A}$ is the activation energy for growth, $T$ the absolute temperature and $R$ the gas constant. In introducing $E_{\Delta}$ it is assumed that the shape of the fibrils and the relation between aggregating area and surface area are independent of the temperature during growth. From the graph of $\log \left(K_{2}\right)_{g}$ against $1 / T$ shown in Fig. 8, $E_{\Delta}=27 \mathrm{kcal} . / \mathrm{mole}$.

Included in Table 3 are data for experiments in which the whole of the precipitations were carried out at different temperatures. Under these conditions:

$$
\frac{\mathrm{d} \ln K_{2}}{\mathrm{~d} 1 / T}=\frac{\mathrm{d} \ln k_{3}}{\mathrm{~d} 1 / T}+\frac{\mathrm{d} \ln n_{e}^{\mathrm{d}}}{\mathrm{d} 1 / T}=-\frac{E_{4}}{R}+\frac{\mathrm{d} \ln \left(r_{e}\right)_{\infty}}{\mathrm{d} 1 / T} .
$$

From the graph of $\log K_{2}$ against $1 / T$ shown in Fig. 8 and the above value of $E_{\Delta}$ the ratio of the radii of the equivalent fibrils $\left(r_{e}\right)_{\infty}$ at $25^{\circ}$ and $37^{\circ}$ is calculated to be 1.6. It can be seen from Fig. 7, which shows the distributions of fibril width at these temperatures, that this figure is of the right order of magnitude.

\section{DISCUSSION}

The satisfactory manner in which the simple twostep mechanism accounts for the shape of the precipitation curves and the fact that the predictions about fibril width are consistent with the electron-microscope data strongly suggest that collagen fibrils form in vitro by the mechanism outlined above, i.e. by the aggregation of soluble collagen particles to form nuclei, followed by the growth of the nuclei into fibrils.

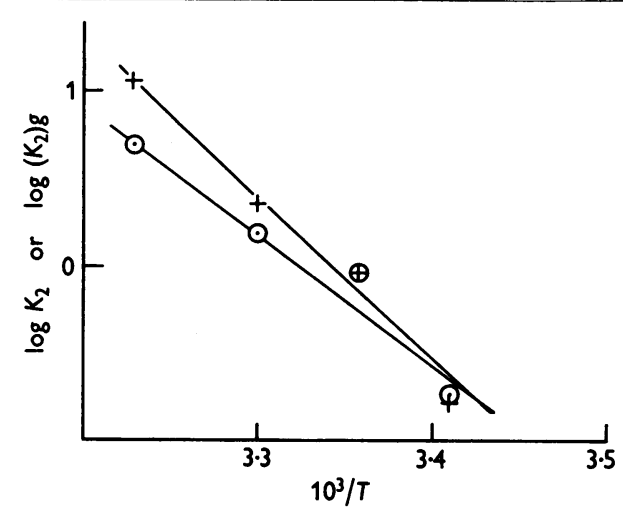

Fig. 8. Variations of $K_{2}(+)$ and $\left(K_{2}\right)_{g}(\odot)$ with absolute temperature, $T$. For definitions of these parameters see text. 
As discussed in the preceding paper, the nature of the soluble collagen particles is uncertain; they may be discrete rod-like collagen molecules or aggregates thereof. The values of the parameter $x$ suggest that two or three of these particles come together to form a nucleus. In view of the asymmetric nature of the collagen molecules the nuclei are probably filamentous; an important point, since the shape as well as the concentration of nuclei plays an important part in determining the rate of precipitation and the size of the ultimate fibrils. The turbidity method used to follow precipitation is, however, unable to give detailed information about nucleation, whose precise nature therefore remains unknown.

The rate of precipitation during the growth phase is determined by the number of fibrils and the rate at which each grows. The rate of the latter process is controlled by the rate of aggregation and by the surface area of the fibrils. Further quantitative electron-microscope observations, particularly at different stages during precipitation, would probably be useful in separating these three factors. It may be noted that the spread of fibril width, as given by equations (10) and (11), should be constant throughout precipitation and this conclusion might also be tested by electron microscopy.

The activation energy for growth under one set of conditions is calculated to be $27 \mathrm{kcal}$./mole. Bensusan \& Hoyt (1958) calculated activation energies for the over-all precipitation process which varied from 23 to $51 \mathrm{kcal} / / \mathrm{mole}$ according to experimental conditions. The activation energy calculated by their method from the present data is about $40 \mathrm{kcal} . / \mathrm{mole}$. If precipitation occurs by the two-step mechanism the precise meaning of Bensusan \& Hoyt's activation energies is obscure, since they are calculated from the complex result of two separate processes. The significance which they attach to the fact that their activation energy is independent of ionic strength in formulating a mechanism for precipitation may not therefore be justified. Bensusan \& Hoyt observed that the maximum slope of graphs of extinction against time were proportional to the square of the collagen concentration. It may be shown that this result follows from the equations for the two-step mechanism if it is assumed that the final distribution of fibril width is independent of collagen concentration: from equation (8)

$$
\left(\frac{\mathrm{d} E}{\mathrm{~d} t}\right)_{\max .}=E_{\infty}\left(\frac{\mathrm{d} p}{\mathrm{~d} t}\right)_{\max .}=E_{\infty} K_{2} C_{0}^{\frac{2}{2}} p_{\max .}^{\frac{3}{2}}\left(1-p_{\text {max. }} .\right) .
$$

It may be shown that $p_{\max .}=0 \cdot 4$, therefore

$$
\begin{aligned}
\left(\frac{\mathrm{d} E}{\mathrm{~d} t}\right)_{\max .}=\text { constant } & \times E_{\infty} K_{2} C_{0}^{\frac{2}{2}} \\
& =\operatorname{constant} \times \frac{E_{\infty} k_{3} C_{0}}{(\rho \pi \alpha)^{\frac{1}{3}}\left(r_{e}\right)_{\infty}} .
\end{aligned}
$$

If $\left(r_{e}\right)_{\infty}$ and hence $E_{\infty} / C_{0}$ are independent of $C_{0}$

$$
\left(\frac{\mathrm{d} E}{\mathrm{~d} t}\right)_{\text {max. }}=\text { constant } \times C_{0}^{2} .
$$

No electron-microscope data are available to test this but since, as described in the preceding paper, the final extinction $\left(E_{\infty}\right)$ of the precipitate is correlated with fibril width, the fact that $E_{\infty} / C_{0}$ varies little with collagen concentration supports the assumption.

In deriving the equation for fibril growth it has been assumed that reaction of the soluble collagen particles at the surface of the precipitate is the rate-controlling step. Several workers (Nielsen 1955, 1958, 1959; Frisch \& Collins, 1953; Collins \& Leinweber, 1956; Ham, 1958) have derived equations for the rate of precipitation when the diffusion of solute to the surface of the precipitate controls the rate and have found that the shape of the precipitation curve is clearly distinguishable from that observed when reaction at the surface is ratecontrolling. If, under certain experimental conditions, diffusion of the soluble collagen particles to the surface of the fibril were the rate-controlling step it should be possible to detect this.

\section{SUMMARY}

1. Fibril formation is regarded as the result of two processes : (a) nucleation, i.e. the aggregation of soluble collagen particles to form nuclei, the smallest aggregates capable of existing as a separate phase; (b) growth of the nuclei into fibrils by accretion of further soluble collagen particles. It is assumed that reaction of the soluble collagen particles with the surface of the growing fibrils controls the rate of growth and that the former process is proportional to the surface area of the precipitate and to the collagen concentration.

2. Equations are derived which qualitatively account for the occurrence of a lag period in precipitation, where the nucleation process predominates over growth, and for the observation that the final distribution of fibril width is determined during the lag period.

3. The equation for growth gives a precise description of the shape of precipitation curves over a wide range of conditions. Predictions about fibril width are consistent with experimental observations.

4. The activation energy for growth is calculated to be $27 \mathrm{kcal}$./mole (at $\mathrm{pH} 7 \cdot 1$, I $0 \cdot 23$, collagen concentration $0.05 \%$ ).

The author wishes to thank Professor R. E. Tunbridge and $\mathrm{Dr} \mathrm{D}$. A. Hall for their interest and encouragement throughout this work. 


\section{REFERENCES}

Anderson, T. F. (1956). In Physical Techniques in Biological Research, vol. 3, p. 178. Ed. by Oster, G. \& Pollister, A. W. New York: Academic Press Inc.

Bensusan, H. B. \& Hoyt, B. L. (1958). J. Amer. chem. Soc. 80, 719 .

Collins, F. C. \& Leinweber, J. P. (1956). J. phys. Chem. 60, 389.

Duke, F. R. \& Brown, L. M. (1954). J. Amer. chem. Soc. 76, 1443.

Frisch, H. L. \& Collins, F. C. (1953). J. chem. Phys. 21, 2158.

Gross, J. (1956). J. biophys. biochem. Cytol. 2, 261.

Gross, J. (1958). J. exp. Med. 107, 265.

Ham, F. S. (1958). J. Phys. Chem. Solids, 6, 335.
Jackson, D. S. (1957). Counc. int. Org. Med. Sci. Symp.: Connective Tissue, p. 69. Ed. by Tunbridge, R. E. Oxford: Blackwell Scientific Publications Ltd.

Johnson, R. A. \& O'Rourke, J. D. (1954). J. Amer. chem. Soc. 74, 2124.

Nielsen, A. E. (1955). J. Colloid Sci. 10, 576.

Nielsen, A. E. (1958). Acta chem. scand. 12, 951.

Nielsen, A. E. (1959). Acta chem. scand. 13, 784.

Schmitt, F. O. (1959). Rev. mod. Phys. 31, 349.

Schmitt, F. O., Gross, J. \& Highberger, J. H. (1955a). Symp. Soc. exp. Biol. 9, 326.

Schmitt, F. O., Gross, J. \& Highberger, J. H. (1955b). Exp. Cell Res. Suppl. 3, 326.

Waugh, D. F. (1957). J. cell. comp. Physiol. 49, Suppl. 1, 145.

Wood, G. C. \& Keech, M. K. (1960). Biochem. J. 75, 588.

Biochem. J. (1960) 75, 605

\title{
The Formation of Fibrils from Collagen Solutions
}

\section{EFFECT OF CHONDROITIN SULPHATE AND SOME OTHER NATURALLY OCCURRING POLYANIONS ON THE RATE OF FORMATION}

\author{
By G. C. WOOD \\ Nuffield Gerontological Research Unit, Department of Medicine, University of Leeds
}

(Received 22 June 1959)

The fact that mucopolysaccharides are abundant in developing connective tissue and the observation that these substances precipitate collagen fibrils from collagen solutions have led to suggestions that they may be important in collagen fibrillogenesis (Meyer, 1946, 1955-56; Gross, 1956).

Except for brief reports by Gross $(1956,1959)$ and Gross \& Kirk (1958) little attention has been paid to the effect of mucopolysaccharides on the kinetics of fibril formation. These workers found that, with two doubtful exceptions, a large number of preparations of chondroitin sulphate, hyaluronic acid, keratosulphate and heparitin sulphate had little or no effect on the rate of fibril formation under the experimental conditions they used. Previous work has shown that the process of collagen-fibril formation may be divided into two phases (Bensusan \& Hoyt, 1958; Wood \& Keech, 1960), which have been termed nucleation and growth (Wood, 1960). In this paper this work is extended to a study of the effect of the presence of low concentrations of certain mucopolysaccharides in the reaction mixture on the kinetics of fibril formation. Particular attention is paid to the effect of chondroitin sulphate on the overall precipitation process and on the growth step alone. It has been suggested (Wood \& Keech, 1960) that different methods of preparing collagen solutions and different methods of pretreating the same collagen solution may result in starting materials, for precipitation experiments, of different degrees of molecular aggregation and therefore having different precipitation properties. A further aim of the present work was therefore to investigate the possibility that the effect of chondroitin sulphate on rate of precipitation depends on the pretreatment of the collagen solution.

The ability to precipitate collagen fibrils is also shown by the nucleic acids (Gross, 1956; Randall, Booth, Burge, Jackson \& Kelly, 1955), which, like the mucopolysaccharides, are polyanionic in nature, and the effect of these substances on the rate of fibril formation has therefore also been investigated.

Part of this work has been reported briefly elsewhere (Wood, 1958).

\section{MATERIALS AND METHODS}

Collagen solutions. For most of the experiments solutions of collagen $(0.1 \%)$ in $0.1 \mathrm{M}-\mathrm{NaCl}$ containing $0.05 \mathrm{M}$-sodium acetate buffer, $\mathrm{pH} 4 \cdot 2$, were prepared from one sample of purified calf dermis (dermis X), as described previously (Wood \& Keech, 1960). For a small number of experiments solutions were prepared in a similar way from another 